\section{Requerimiento de servicios hospitalarios en Girugía Estêtica ambulatoria: análisis de complicaciones en 13.411 casos consecutivos e implicaciones para la práctica en el contexto de la pandemia COVID-19}

\section{Inhospital treatment requirements in ambulatory Aesthetic Surgery: complications analysis of $13.411 \mathrm{cases}$ and surgical practice repercussions for COVID-19 pandemic}

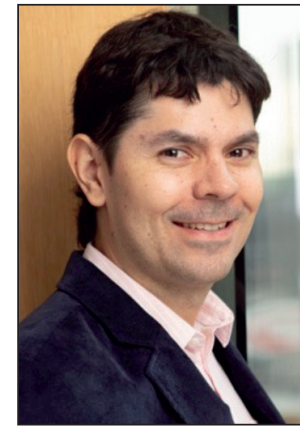

López Rios A.A.
Adolfo Alejandro LÓPEZ RIOS*, Alfredo Salvador PATRÓN GÓMEZ*, Juan Carlos VÉLEZ LARA**
Resumen

Introducción y objetivo. La Cirugía Plástica Estética se ha visto suspendida en muchas regiones del mundo debido a la pandemia de la pacientes afectados gravemente por esta enfermedad y la morbilidad atribuible a la realización inadvertida de un acto quirúrgico optativo en un paciente con infección asintomática, así como el riesgo de contagio bidireccional en instituciones de salud, soportan las cancelaciones masivas de procedimientos con fines estéticos. La reactivación de estos servicios debe considerar e balance riesgo/beneficio con las diferentes fases de la pandemia y la epidemiología local. Asumiendo la reapertura de los servicios de Cirugía Estética es posible plantear que un número importante de procedimientos estéticos tienen riesgos bajos de complicaciones y podrían ser susceptibles de realizarse de manera segura.

Material y método. Estudio descriptivo y retrospectivo en pacientes intervenidos en un centro de Cirugía Estética ambulatorio que requirieron atención médica por complicaciones asociadas en un período pre COVID-19 de 36 meses, con el fin de identificar variables y frecuencia de complicaciones susceptibles de comprometer recursos médicos hospitalarios adicionales.

Resultados. En 3 años se realizaron 13.411 cirugías estéticas. El $67.5 \%$ de los procedimientos fue único y en el $32.5 \%$ se realizaron 2 o más procedimientos en el mismo acto anestésico. Se presentaron 244 complicaciones en los primeros 30 días posoperatorios, para una tasa de $1.8 \%$ de complicaciones en 3 años. De estos, el tiempo quirúrgico promedio fue de $200 \mathrm{mi}-$ nutos, 44 pacientes (18\%) fueron manejados en hospital y $200(82 \%)$ como ambulatorios.

Conclusiones. Se sugiere que el restablecimiento de los servicios de cirugías electivas estéticas en el contexto COVID-19 debería iniciar con cirugías únicas, tiempos cortos de duración y menores a 2 horas, con el fin de disminuir la necesidad de recursos hospitalarios adicionales.

Palabras clave COVID-19, Complicaciones posoperatorias, Cirugía Plástica, Cirugía Estética, Cirugía ambulatoria.

\section{Nivel de evidencia científica Recilinio [esta versión] ABentado} 4c Terapéutico COVID-19. La preservación de recursos hospitalarios para la atención de los

Background and objective. Aesthetic Plastic Surgery has been suspended in many regions of the world due to the COVID-19 pandemic. The preservation of available hospital resources for the care of patients seriously affected by this disease and the morbidity attributable to the inadvertent performance of an optional surgical act in a patient with asymptomatic infection, as well as the risk of bidirectional infection in health institutions, support massive cancellations of procedures with aesthetic fines. The reactivation of these services must consider the risk/benefit balance with the different phases of the pandemic and local epidemiology. Assuming the reopening of Aesthetic Surgery services, it is possible to propose a significant number of aesthetic procedures that have low risks of complications, and may be capable of being treated safely.

Methods. Descriptive and retrospective study in patients operated on in an outpatient Aesthetic Surgery center, who required medical attention for associated complications in a previous COVID-19 period of 36 months, in order to identify variables and frequency of complications susceptible to commit additional hospital medical resources.

Results. In a 3-year period, a total of 13.411 aesthetic surgeries were performed; $67.5 \%$ of the procedures were unique and in $32.5 \%$ two or more procedures were processed in the same anesthetic act. In the first 30 postoperative days, 244 complications were observed for a $1.8 \%$ complication rate in 3 years. At the moment, the average surgical time was 200 minutes, 44 patients $(18 \%)$ were managed at the hospital level and $200(82 \%)$ were ambulatory.

Conclusions. Consider that the reestablishment of elective Aesthetic Surgery services in the COVID-19 context it should start with single surgeries, short times of duration and less than 2 hours, in order to decrease the need for additional hospital resources.

\section{Key words COVID-19, Postoperative complications, Plastic Surgery, Aesthetic Surgery, Ambulatory surgical procedures.}

\section{Level of evidence Received [this version] Accepted}

$4 c$ Therapeutic July 20 / 2020 May $16 / 2021$

Conflicto de intereses: Los autores declaran no tener ningún interés financiero relacionado con el contenido de este artículo.

Financiación: No hubo fuentes externas de financiación para este trabajo. 
Introducción

La enfermedad causada por el virus SARS-CoV-2 era desconocida antes del brote originado en Wuhan (China) en diciembre de 2019. Actualmente la COVID-19 es una pandemia cuyos síntomas más comunes son fiebre, tos seca, debilidad, fatiga, cefalea, congestión nasal, conjuntivitis, odinofagia, diarrea, perdida del gusto y el olfato, además de brotes cutáneos principalmente en manos y pies. Estos síntomas pueden ser leves en la mayoría de los infectados y hasta el $80 \%$ se recupera sin requerir tratamiento hospitalario. Sin embargo, 1 de cada 5 pacientes experimenta síntomas respiratorios graves, especialmente aquellas poblaciones con factores de riesgo como edad avanzada, hipertensión, comorbilidades cardiorrespiratorias, obesidad, diabetes, cáncer o inmunosupresión, que tienen mayor posibilidad de presentar síntomas graves y fallecer a consecuencia de esta enfermedad. Los casos más severos requieren atención hospitalaria, incluyendo cuidados intensivos, que aumentan la presión sobre los sistemas sanitarios, de tal forma que se ven forzados a adoptar planes de contingencia para utilizar de manera eficiente los recursos existentes con el fin de preservar el mayor número de vidas. ${ }^{(1)}$

Al tratarse de una pandemia que evoluciona cada día, las estadísticas cambian constantemente, con varios millones de personas infectadas y varios centenares de fallecidos en múltiples países del mundo. La atención a la COVID-19 ha sobrepasado la capacidad de respuesta de muchos sistemas de salud, demandando la implementación de cambios drásticos en los modelos de atención buscando optimizar los recursos y aumentar las capacidades hospitalarias y de las unidades de cuidados intensivos. Las acciones desarrolladas por los gobiernos incluyen el cierre de fronteras, el distanciamiento social, cuarentenas de ciudades y países, cierre de instituciones académicas, suspensión del comercio y limitación de las actividades hospitalarias a solo aquellas urgentes, afectando directamente a la práctica de la Cirugía Plástica Estética con la cancelación de todo tipo de intervenciones quirúrgicas electivas.

Entre los argumentos que motivan la restricción de estas cirugías electivas se encuentra el estimado aumento de la morbimortalidad que pueden conllevar en pacientes con la enfermedad causada por el virus SARS-CoV-2 en fase asintomática, la necesidad de mantener recursos hospitalarios disponibles, la posibilidad de infección por el virus en el personal de salud, la limitada disponibilidad de reactivos o pruebas diagnósticas para la COVID-19 y la carencia de elementos de protección personal en algunas regiones, considerando que las cirugías estéticas pueden posponerse sin impactar directamente en la salud física del paciente.
Estos cambios han generado una crisis sin precedentes en la práctica de la Cirugía Plástica Estética. Muchos servicios de Cirugía Estética ambulatoria se encuentran cerrados o limitados en sus funciones debido a la pandemia. Entre las ventajas de las unidades quirúrgicas ambulatorias se encuentra el mayor control a la hora de programar las cirugías electivas, aumento en la privacidad, el confort en el lugar de atención y mejor costo-eficiencia en relación con el entrenamiento y preparación del equipo médico, enfermería y personal de soporte. En los Estados Unidos, 4 de cada 5 procedimientos estéticos $(80 \%)$ se realizan en ambientes ambulatorios. El uso de medicamentos anestésicos de rápida acción y el estrecho monitoreo perioperatorio de los pacientes ha demostrado ventajas en términos de velocidad de recuperación posoperatoria, menores efectos adversos, costos y duración de la anestesia, por lo cual es común la realización de la Cirugía Estética en centros ambulatorios. ${ }^{(2-4)}$ Estudios previos han reportado entre un $0.7 \%$ y un $1 \%$ en la frecuencia de complicaciones, necesidad de reintervención y requerimiento de hospitalización en los servicios de cirugía ambulatoria, siendo este tipo de pacientes con complicaciones quienes podrían entrar a competir por recursos e insumos necesarios para la atención de los pacientes afectados severamente por el coronavirus SARS$\mathrm{CoV}-2 \cdot{ }^{(5,6)}$

Es necesario redefinir la práctica de la Cirugía Plástica Estética asumiendo que, a corto plazo, la COVID-19 no desaparecerá. La nueva normalidad precisa desarrollar nuestra actividad quirúrgica en convivencia con esta enfermedad. El consumo posoperatorio de salas de hospitalización, hemoderivados, laboratorio o cuidados intensivos son críticas variables en el contexto de la pandemia. El presente estudio pretende identificar de manera objetiva las características y el tipo de procedimientos de Cirugía Plástica Estética que no requieren recursos adicionales, analizando una base de datos recolectada antes de la aparición de la enfermedad causada por el virus SARS-CoV-2 en un centro de atención ambulatoria.

\section{Material y método}

Realizamos un estudio descriptivo y retrospectivo con base en las historias clínicas de los pacientes que requirieron atención médica debido a complicaciones después de someterse a una intervención quirúrgica de Cirugía Estética, operados en un centro de atención ambulatoria de baja y mediana complejidad con énfasis en Cirugía Plástica de Medellín (Colombia). El periodo de estudio definido fue de 36 meses, entre el primero de enero de 2009 y el 31 de diciembre de 2011, fase en la cual la institución no contaba con servicio de hospitalización. 
La información fue recolectada por el autor principal en Microsoft Excel 365 y el análisis realizado fue de tipo corte. Incluimos todos los pacientes sometidos a procedimientos quirúrgicos de carácter estético realizados por los cirujanos plásticos que tenían su práctica en la institución en este período de tiempo y que requirieron algún tipo de atención médica asociada a complicación quirúrgica o anestésica en los primeros 30 días de posoperatorio. También todas las complicaciones presentadas que requirieron observación posoperatoria, hemocomponentes, reintervención quirúrgica y hospitalización o cuidados intensivos.

Una vez recolectada la información, desarrollamos un análisis estadístico utilizando el software Stata 14.1 para la descripción de las variables de tipo cualitativo utilizando distribuciones de frecuencias y gráficos de barras. Describimos las variables de tipo cuantitativo mediante medidas de tendencia central y de dispersión.

El proyecto fue presentado y aprobado por el Comité de Ética del centro (IQ Interquirófanos, Medellín, Colombia) y avalado por el Comité de Investigación de la Sección de Cirugía Plástica, Maxilofacial y de la Mano de la Universidad de Antioquia (Medellín, Colombia).

\section{Resultados}

Durante el período de estudio analizamos 13.411 cirugías: 12.472 (93\%) en mujeres y 939 (7\%) en hombres. En el $67.5 \%(n=9055)$ se realizó un solo procedimiento y en el $32.5 \%(n=4356)$ dos o más procedimientos. Durante este periodo, la institución IQ Interquirófanos contaba únicamente con servicios de cirugía ambulatoria, sin sala de hospitalización, por lo cual los pacientes que requerían manejo hospitalario eran trasladados a otro centro asistencial. Esta característica fue de suprema importancia para elegir el período de tiempo a tomar de la base de datos para la recolección de la información, minimizando sesgos en el manejo de complicaciones y asumiendo, como sucede actualmente, la autodependencia como centro ambulatorio para evitar consumo de recursos del sistema de salud (Tabla I).

Tabla I. Cirugías realizadas en el período de estudio y según procedimiento único o combinado.

\begin{tabular}{|l|c|c|c|c|c|}
\hline & $\mathbf{2 0 0 9}$ & $\mathbf{2 0 1 0}$ & $\mathbf{2 0 1 1}$ & Total & Porcentaje \\
\hline $\begin{array}{l}\text { Un solo } \\
\text { procedimiento } \\
\text { por cirugía }\end{array}$ & 2503 & 3268 & 3284 & 9055 & $67.50 \%$ \\
\hline $\begin{array}{l}\text { Dos o más } \\
\text { procedimientos } \\
\text { por cirugía }\end{array}$ & 1773 & 1203 & 1380 & 4356 & $32.50 \%$ \\
\hline Total & $\mathbf{4 2 7 6}$ & $\mathbf{4 4 7 1}$ & $\mathbf{4 6 6 4}$ & $\mathbf{1 3 4 1 1}$ & $\mathbf{1 0 0 \%}$ \\
\hline
\end{tabular}

La edad media de los pacientes que presentaron complicaciones en los primeros 30 días de posoperatorio fue de 36 años (desviación estándar de 12 años, percentil 25 fue 26 años y percentil 75 de 44 años).

El tiempo quirúrgico en promedio de los pacientes que presentaron complicaciones mayores en los primeros 30 días fue de 200 minutos (desviación estándar de 104 minutos, percentil 50 de 195 minutos, percentil 25 de 113 minutos y percentil 75 de 270.5 minutos).

En total, 244 actos quirúrgicos presentaron complicaciones en los primeros 30 días de posoperatorios, en su mayoría en cirugías combinadas con 2 o más procedimientos para una tasa del $1.8 \%$ de complicaciones en 3 años; de estas el $93.85 \%(n=229)$ fueron en mujeres y el $6.15 \%(n=15)$ en hombres.

De las 244 cirugías realizadas que presentaron complicaciones en el periodo estudiado el $34.8 \%(n=85)$ de los casos se realizó un único procedimiento y en el 65.2\% $(n=159)$ se realizaron 2 más procedimientos en el mismo acto quirúrgico (Tabla II). En cuanto a la distribución según el tipo de procedimiento, el número total de cirugías realizadas fue de 13.411 , de las que $4.356(32,5 \%)$ fueron cirugías combinadas (en un solo acto anestésico o cirugía se realizaron varios procedimientos).

Tabla II. Complicaciones presentadas según procedimiento único o combinado.

\begin{tabular}{|l|c|c|}
\hline Procedimiento & Número & Porcentaje \\
\hline Único & 85 & 34.8 \\
\hline Combinado & 159 & 65.2 \\
\hline Total & 244 & 100 \\
\hline
\end{tabular}

Entre los pacientes que presentaron complicaciones en el período estudiado, $44(18 \%)$ requirieron tratamiento intrahospitalario y $200(82 \%)$ fueron tratados de forma ambulatoria (Tabla III). El tratamiento intrahospitalario en 32 casos (72.7\%) consistió en estudios adicionales y manejo médico; 7 pacientes (15.9\%) fueron admitidos para tratamiento de hipovolemia, 4 pacientes $(9.1 \%)$ presentaron dificultad respiratoria posoperatoria y se hospitalizaron con sospecha de tromboembolismo pulmonar, y 1 caso $(2.3 \%)$ presentó dolor abdominal por lo que fue remitido a un centro de mayor complejidad para estudio y seguimiento (Tabla IV y V).

Tabla III. Distribución del manejo hospitalario o ambulatorio de los pacientes que presentaron complicaciones.

\begin{tabular}{|l|c|c|}
\hline Lugar de atención & Número & Porcentaje \\
\hline Hospitalización & 44 & $18 \%$ \\
\hline Ambulatorio & 200 & $82 \%$ \\
\hline Total & $\mathbf{2 4 4}$ & $\mathbf{1 0 0} \%$ \\
\hline
\end{tabular}


Tabla IV. Distribución según causa de hospitalización entre los pacientes que presentaron complicaciones.

\begin{tabular}{|l|c|c|}
\hline Causa hospitalización & Número & Porcentaje \\
\hline $\begin{array}{l}\text { Vigilancia posoperatoria/estudio } \\
\text { intrahospitalario }\end{array}$ & 32 & $72.7 \%$ \\
\hline Hipovolemia & 7 & $15.9 \%$ \\
\hline Dificultad respiratoria & 4 & $9.1 \%$ \\
\hline Abdomen agudo & 1 & $2.3 \%$ \\
\hline Total & $\mathbf{4 4}$ & $\mathbf{1 0 0} \%$ \\
\hline
\end{tabular}

Documentamos 18 pacientes con complicaciones tras ritidoplastia, de los cuales $12(66.6 \%)$ fueron por hematoma, $3(16.6 \%)$ por dehiscencia de heridas y 1 por cada una de las siguientes causas: broncoaspiración, evento adverso a medicamento y anemia aguda asociada a cirugía combinada.

Con respecto a las complicaciones de abdominoplastia, identificamos 29 pacientes (50.8\%) con anemia aguda, todos con liposucción como cirugía adicional. Documentamos hematomas en 9 (15.7\%), dehiscencias en $7(12.3 \%)$, seromas en $4(7 \%)$ y necrosis del colgajo abdominal en $2(3.5 \%)$. Otras complicaciones incluyeron trastorno ácido-básico, síndrome coronario agudo, tromboembolismo pulmonar, trombosis venosa profun$\mathrm{da}$, trastorno visual posoperatorio y efecto adverso a medicamentos, con 1 caso de cada una.

De los 136 pacientes complicados tras liposucción, la mayoría, $121(89 \%)$ fueron sometidos a procedimientos combinados y $15(11 \%)$ a procedimiento único (Tabla VI). De estas complicaciones asociadas a liposucción, el 43\% $(\mathrm{n}=58)$ fueron por anemia aguda. Al tratarse de cirugías
Tabla VI. Complicaciones por liposucción distribuidas en procedimiento único o combinado.

\begin{tabular}{|l|c|c|}
\hline $\begin{array}{l}\text { Complicaciones por } \\
\text { liposucción }\end{array}$ & Número & Porcentaje \\
\hline Procedimiento único & 15 & $11 \%$ \\
\hline Procedimiento combinado & 121 & $89 \%$ \\
\hline Total & $\mathbf{1 3 6}$ & $\mathbf{1 0 0 \%}$ \\
\hline
\end{tabular}

combinadas, identificamos hematomas en 19 pacientes (14\%), dehiscencia en la cirugía abdominal o mamaria en 14 pacientes $(10 \%)$, seroma en 14 pacientes $(10 \%)$, infección del sitio operatorio en 10 pacientes (7\%), edema agudo de pulmón o depresión respiratoria en 8 pacientes $(6 \%), 2$ pacientes $(1.5 \%)$ con necrosis del colgajo de abdominoplastia, 2 (1.5\%) con trombosis venosa profunda y $2(1.5 \%)$ con tromboembolismo pulmonar. Además, 1 paciente en cada una de las siguientes causas: lesión intestinal, trastorno visual posoperatorio, cuerpo extraño dejado en cirugía (específicamente en la cirugía combinada con mastopexia), síndrome coronario agudo, hemorragia posoperatoria, isquemia cerebral transitoria y fallecimiento.

En la mamoplastia de aumento con implantes identificamos 109 complicaciones en 3 años; 44 pacientes presentaron hematomas (40\%), seguidos de anemia aguda en 19 pacientes $(17 \%)$ en procedimientos combinados, especialmente liposucción. Otras complicaciones de la mamoplastia de aumento incluyeron: seroma en 13 pacientes $(12 \%)$, dehiscencia en 8 pacientes $(7.3 \%)$, infec-

Tabla V. Distribución de complicaciones por año y tipo de procedimiento*

\begin{tabular}{|c|c|c|c|c|c|c|}
\hline Procedimientos & 2009 & 2010 & 2011 & Complicaciones & Procedimientos totales & \% Complicaciones \\
\hline Ritidoplastia & 103 & 103 & 90 & 18 & 296 & $6.08 \%$ \\
\hline Abdominoplastia & 352 & 426 & 388 & 57 & 1166 & $4.88 \%$ \\
\hline Liposucción & 1426 & 1534 & 1395 & 136 & 4355 & $3.12 \%$ \\
\hline $\begin{array}{l}\text { Mamoplastia } \\
\text { aumento con } \\
\text { implantes }\end{array}$ & 1465 & 1694 & 1533 & 109 & 4692 & $2.32 \%$ \\
\hline $\begin{array}{l}\text { Mastopexia y } \\
\text { mamoplastia de } \\
\text { reducción }\end{array}$ & 468 & 553 & 652 & 33 & 1673 & $1.97 \%$ \\
\hline Blefaroplastia & 337 & 311 & 324 & 18 & 927 & $1.94 \%$ \\
\hline Rinoseptoplastia & 310 & 355 & 346 & 17 & 1011 & $1.68 \%$ \\
\hline
\end{tabular}

*Distribución según tipo de procedimiento. El numero total de cirugías realizadas fue de 13.411, de estas, 4.356 (32.5\%) fueron cirugías combinadas, es decir en un solo acto anestésico o cirugía se realizaron varios procedimientos. 
ción del sitio operatorio en 7 pacientes $(6.4 \%)$, necrosis del complejo areola-pezón en 3 pacientes (2.7\%), edema agudo de pulmón/depresión respiratoria en 3 pacientes (2.7\%), evento adverso asociado a medicamentos en 3 pacientes $(2.7 \%)$. Además 1 caso de cada una de las siguientes causas: laringoespasmo, herida intestinal (en cirugía combinada con liposucción), sinmastia o asimetría, neumotórax, trastorno acido-base, quemadura, contractura capsular, tromboembolismo pulmonar y síndrome coronario (en cirugía combinada).

En las mastopexias y mamoplastias de reducción registramos 33 pacientes con complicaciones: 13 (39.3\%) con hematomas, 5 (15\%) con anemia aguda, en combinación con liposucción especialmente. Identificamos además dehiscencia en 4 pacientes (12\%), infección del sitio operatorio en $4(12 \%)$, necrosis del complejo areola-pezón en 3 pacientes ( $9 \%$ ) y 1 caso de cada una de las siguientes causas: edema agudo de pulmón o depresión respiratoria, seroma, contractura capsular y cuerpo extraño dejado en cirugía.

En la blefaroplastias identificamos 18 pacientes con complicaciones, 8 (44\%) con hematomas en cirugías combinadas y 1 por cada una de las siguientes complicaciones: infección del sitio operatorio, broncoaspiración (en cirugía combinada con ritidoplastia), anemia aguda (en cirugía acompañada de liposucción), evento adverso a medicamentos, trombosis venosa profunda (en cirugía combinada con lipoabdominoplastia), úlcera corneana, hematoma retrobulbar y 1 fallecimiento en cirugía combinada con liposucción, lipoinyección glútea y corrección de cicatriz.

Registramos 17 pacientes con complicaciones tras rinoplastia, 5 con hematomas $(29 \%)$ en cirugías combinadas con ritidoplastia o cirugía corporal, 3 (17\%) con anemia aguda en cirugía combinada con liposucción y 2 (12\%) con laringoespasmo. Otras complicaciones incluyeron: hemorragia posoperatoria (en cirugía combinada con liposucción), evento adverso a medicamento y edema agudo de pulmón/depresión respiratoria, con 1 caso cada una. Además, 2 pacientes de cirugía combinada de rinoplastia y cirugía mamaria que presentaron dehiscencia y necrosis del complejo areola-pezón.

Al examinar la distribución absoluta según la frecuencia del tipo de complicación sin considerar el tipo de cirugía, encontramos que el hematoma fue la complicación con mayor frecuencia con 79 casos (31.9\%), seguido de la anemia aguda con 64 casos $(25.8 \%)$. Los seromas se presentaron en 26 casos (10.5\%), la infección del sitio operatorio en 24 casos (9.7\%), la dehiscencia en 21 casos (8.5\%), la necrosis del complejo areola-pezón/colgajos en 10 casos (4\%), la depresión respiratoria posoperatoria o el edema agudo de pulmón en 10 casos (4\%), eventos adversos a medicamentos en 8 casos (3.2\%), laringoespasmo posoperatorio en 4 casos $(1.6 \%)$ y trombosis venosa profunda en miembros inferiores con 2 casos $(0.8 \%)$ (Tabla VII). Cuatro pacientes presentaron 2 complicaciones tras cirugía: hematoma y anemia aguda.

$\mathrm{Al}$ analizar los resultados encontramos asociación entre la liposucción y la presencia de anemia y hematomas que requirió transfusión. La mayoría de las complicaciones se presentaron cuando los procedimientos fueron combinados, con un valor $\mathrm{p}<0.05$.

Las complicaciones isquémicas y embólicas fueron poco frecuentes y cuando se presentaron se asociaron con abdominoplastia y liposucción.

Después de un procedimiento combinado de $275 \mathrm{mi}-$ nutos de duración que incluyó liposucción, lipoinyección, blefaroplastia y resección de cicatriz, se produjo 1 fallecimiento (la paciente desarrolló un cuadro anémico agudo progresivo que requirió múltiples transfusiones, reintervención quirúrgica con laparotomía exploradora sin encontrar lesión intraabdominal o retroperitoneal, seguida de shock hipovolémico asociado a coagulopatía).

El tratamiento de las complicaciones consistió en drenaje quirúrgico en 93 casos (38.1\%); de estos, sólo 7 pacientes continuaron tratamiento intrahospitalario. Se requirió hemotransfusión en 54 pacientes $(22.1 \%)$ de los

Tabla VII. Distribución según frecuencia absoluta y porcentaje de complicaciones.

\begin{tabular}{|c|c|c|}
\hline Complicación & $\begin{array}{c}\text { Frecuencia } \\
\text { absoluta }\end{array}$ & Porcentaje \\
\hline Hematoma* & 79 & $31.9 \%$ \\
\hline Anemia aguda* & 64 & $25.8 \%$ \\
\hline Seroma & 26 & $10.5 \%$ \\
\hline $\begin{array}{l}\text { Infección sitio } \\
\text { operatorio }\end{array}$ & 24 & $9.7 \%$ \\
\hline Dehiscencia & 21 & $8.5 \%$ \\
\hline $\begin{array}{l}\text { Necrosis complejo } \\
\text { areola-pezón/ } \\
\text { necrosis colgajos }\end{array}$ & 10 & $4.0 \%$ \\
\hline $\begin{array}{l}\text { Edema agudo de } \\
\text { pulmón/depresión } \\
\text { respiratoria }\end{array}$ & 10 & $4.0 \%$ \\
\hline $\begin{array}{l}\text { Evento adverso } \\
\text { medicamento }\end{array}$ & 8 & $3.2 \%$ \\
\hline Laringoespasmo & 4 & $1.6 \%$ \\
\hline $\begin{array}{l}\text { Trombosis } \\
\text { venosa profunda } \\
\text { en miembros } \\
\text { inferiores }\end{array}$ & 2 & $0.8 \%$ \\
\hline Total & 248 & $100 \%$ \\
\hline
\end{tabular}

* Cuatro pacientes presentaron 2 complicaciones después de cirugía: hematoma y anemia aguda, total de cirugías 244. 
Tabla VIII. Distribución según tipo de tratamiento de las complicaciones.

\begin{tabular}{|l|c|c|}
\hline $\begin{array}{l}\text { Tratamiento de la } \\
\text { complicación }\end{array}$ & Número & Porcentaje \\
\hline Drenaje quirúrgico & 93 & $38.1 \%$ \\
\hline Hemotransfusión & 54 & $22.1 \%$ \\
\hline $\begin{array}{l}\text { Seguimiento } \\
\text { ambulatorio/ } \\
\text { medicamentos }\end{array}$ & 30 & $12.3 \%$ \\
\hline Reintervención & 26 & $10.7 \%$ \\
\hline Hospitalización & 18 & $7.4 \%$ \\
\hline $\begin{array}{l}\text { Soporte respiratorio } \\
\text { posoperatorio }\end{array}$ & 8 & $4.9 \%$ \\
\hline $\begin{array}{l}\text { Lavado, } \\
\text { desbridamiento } \\
\text { quirúrgico, retirada } \\
\text { de prótesis }\end{array}$ & 2 & $3.3 \%$ \\
\hline $\begin{array}{l}\text { Laparotomía } \\
\text { exploradora }\end{array}$ & $\mathbf{2 4 4}$ & $\mathbf{1 0 0 \%}$ \\
\hline Toracostomía & & $0.8 \%$ \\
\hline Total & $12 \%$ \\
\hline
\end{tabular}

que 15 continuaron tratamiento intrahospitalario. Se realizó seguimiento ambulatorio en 30 pacientes (12.3\%), reintervención diferente a drenaje de hematoma en 26 casos $(10.7 \%)$, soporte respiratorio posoperatorio en 12 pacientes $(4.9 \%)$, de los cuales 1 requirió hospitalización. Se realizó lavado, desbridamiento y/o retirada de prótesis en 8 pacientes $(3.3 \%)$, laparotomía exploradora en 2 pacientes $(0.8 \%)$, toracostomía en 1 paciente $(0.4 \%)$ que continúo tratamiento intrahospitalario; 18 pacientes (7.4\%) requirieron hospitalización para estudio, seguimiento y control posoperatorio.

De los pacientes con complicaciones, 44 fueron admitidos a hospitalización (18\%), de los cuales 32 (73\%) fueron hospitalizados para realización de estudios diagnósticos y seguimiento posoperatorio, 7 (16\%) por hipovolemia sintomática, 4 por dificultad respiratoria o hipoxemia y $1(2 \%)$ por dolor abdominal. Dos pacientes fueron identificados con trombosis venosa profunda (Tabla VIII).

El análisis determina que si se quisiera disminuir el número de pacientes que requieren hospitalización después de una intervención de Cirugía Estética, objetivamente los procedimientos que más aportan a estas cifras son las abdominoplastias y liposucciones. Las complicaciones de cirugías mamarias y faciales en su gran mayoría son susceptibles de manejo en el centro de atención ambulatoria sin requerir hospitalización.

Durante nuestro periodo de estudio de 3 años identificamos 244 complicaciones sobre un total de 13.411
Tabla IX. Asociación entre liposucción y complicaciones comparada con otros procedimientos.

\begin{tabular}{|l|c|c|c|}
\hline & $\begin{array}{c}\text { Expuestos a } \\
\text { liposucción }\end{array}$ & $\begin{array}{c}\text { No expuestos } \\
\text { a liposucción }\end{array}$ & \\
\hline $\begin{array}{l}\text { Casos } \\
\text { Complicados }\end{array}$ & 127 & 117 & 244 \\
\hline No complicados & 3571 & 9596 & 13167 \\
\hline Total & $\mathbf{3 6 9 8}$ & $\mathbf{9 7 1 3}$ & $\mathbf{1 3 4 1 1}$ \\
\hline
\end{tabular}

pacientes operados. La asociación entre liposucción y complicaciones en comparación con otros procedimientos resultó en una Odds ratio de 2.91 (95\% CI 2.26-3.75) y la prueba exacta de Fisher con un valor de $p<0.0001$ (Tabla IX).

\section{Discusión}

La pandemia de la COVID-19 ha tenido repercusiones globales con impacto claro en todas las áreas de la salud y la economía. La Cirugía Plástica Estética, al tratarse de un servicio de salud electivo, se ha visto afectada al cerrar de forma temporal las salas de cirugía con el fin de mantener el aislamiento social y sobre todo para centrar los recursos hospitalarios y médicos en la atención de pacientes afectados por el coronavirus. Después de varios meses de pandemia, cierre de la economía y políticas instauradas para mitigar la propagación de la enfermedad, son necesarias estrategias para la reapertura gradual de los servicios quirúrgicos ambulatorios electivos. Las cirugías a tener en cuenta en este proceso serían aquellas que impliquen el menor requerimiento de servicios hospitalarios adicionales. Por esto, consideramos importante reconocer en un Servicio de Cirugía Plástica ambulatoria, de manera objetiva y en una etapa previa a la COVID-19, las características de los procedimientos de aquellas intervenciones de Cirugía Estética electiva ambulatoria que requirieron recursos adicionales debido a complicaciones, especialmente aquellas con necesidad de hospitalización y remisión a instituciones de mayor complejidad. Al identificar estadísticamente las complicaciones y los tipos de cirugías con menores requerimientos hospitalarios en el posoperatorio, podríamos contribuir en la decisión individual del tipo de cirugías electivas estéticas practicables en la fase inicial de la reapertura de estos servicios o durante los posibles períodos de rebrotes posteriores.

A pesar de que los procedimientos de Cirugía Plástica Estética tienen un balance riesgo-beneficio favorable, se realizan por lo general en población sin comorbilidades $\mathrm{y}$ con pocos factores de riesgo y son diferibles, hay que 
tener en cuenta que consumen recursos sanitarios y existe la posibilidad de que se lleven a cabo en pacientes con infección inadvertida por SARS-CoV-2, asintomáticos y con el riesgo de contaminación en doble vía en las instituciones de salud, todo lo cual hace parte de las consideraciones tenidas en cuenta por las autoridades sanitarias para restringir este tipo de cirugías estéticas durante las fases más activas de la pandemia.

Sin embargo, también es cierto que muchos procedimientos estéticos que presentan complicaciones son resueltos en los mismos centros de cirugía ambulatoria, sin requerir recursos hospitalarios adicionales de la red de salud. Entre las múltiples complicaciones descritas en Cirugía Estética están la aparición de hematomas, la infección del sitio operatorio superficial, profunda o de órgano o espacio, anemia, dehiscencia de la herida, necrosis cutánea y seromas, entre otros. Otras más serias incluyen trombosis venosa profunda, tromboembolismo pulmonar, embolismo graso, hipovolemia, complicaciones anestésicas y por hemotransfusión, neumonía, fallo cardiaco, infarto de miocardio, edema pulmonar, toxicidad por anestésicos locales y generales, enfermedad cerebrovascular, isquemia cerebral transitoria, lesiones vasculares e intraabdominales y fallecimiento. En nuestro estudio, identificamos las complicaciones en el primer mes de posoperatorio y que requirieron atención ambulatoria, urgente y hospitalaria. La mayoría de estas complicaciones identificadas fueron manejadas sin requerir hospitalización o recursos médicos que en el momento deben ser destinados para pacientes con COVID-19.

El porcentaje de complicaciones que hallamos fue de 244 sobre un total de 13.411 pacientes intervenidos en un período de 3 años, con una tasa del 1.8\%. Se presentó un solo fallecimiento durante este período. Entre los pacientes que presentaron complicaciones, documentamos hematoma en el $32 \%$, anemia aguda en el $26 \%$, seromas en el 10\%, infección del sitio operatorio en el 10\% y dehiscencia en el $8 \%$. Nuestros hallazgos se correlacionan con múltiples reportes, como el estudio realizado por Byrd y col. ${ }^{(7)}$ con 5.316 cirugías, en su mayoría estéticas, realizadas en un centro quirúrgico ambulatorio de baja complejidad en la ciudad de Dallas, Texas, Estados Unidos, durante un período de 6 años, quienes describen un total de 35 complicaciones correspondientes al $0.7 \%$, sin fallecimientos durante ese tiempo. La mayoría de las complicaciones se debían a hematomas, con un 77\% de todos los casos. Las infecciones posoperatorias se presentaron en el $0.52 \%$ del total de pacientes y en el $0.11 \%$ obligaron a llevar al paciente al quirófano para incisión y drenaje. En total, 7 pacientes requirieron tratamiento intrahospitalario después del procedimiento quirúrgico por arritmias, angina y embolia pulmonar. La combinación de procedimientos representó el 10.2\% de los casos y no se atribuyeron resultados adversos por esta causa. Datos adicionales reportados por Teimourian ${ }^{(8)}$ por medio de una encuesta por correo realizada a todos los miembros de la Sociedad Americana de Cirugía Plástica y Reconstructiva, determinaron una tasa de complicaciones en liposucción del $0.1 \%$, en dermolipectomía del $0.9 \%$ y en abdominoplastia del 2\%. El total de procedimientos reportados fue de 112.756 , de los cuales fueron reportados 15 fallecimiento, 2 secundarios a liposucción, 2 a dermolipectomía y 11 a abdominoplastia. El tromboembolismo pulmonar fue la causa del fallecimiento en el $60 \%$ de los casos (9 pacientes). Además, Rubin y Matarasso ${ }^{(9)}$ reportaron una mortalidad de entre 1 en cada 100.000 a 1 en cada 300.000. La mortalidad en las abdominoplastias se presentó en el 41.4 por cada 100.000 procedimientos. En pacientes sometidos a liposucción, el tromboembolismo pulmonar se presentó en 33.1 por cada 100.000 y las complicaciones anestésicas se presentaron en 30.4 por cada $100.000 .{ }^{(8)}$ Las complicaciones secundarias a lipoplastia fluctúan en un rango entre $0.3 \%$ y $1.8 \%$. Según Roje, ${ }^{(10)}$ el riesgo de una complicación severa, incluyendo el fallecimiento, después de uns abdominoplastia fluctúa entre 1 en 617 y 1 en 2.320 casos. . $^{(1,12)}$

La infección del sitio operatorio se presentó en 24 de los 13.411 pacientes operados en un periodo de 3 años, con una tasa del $0.1 \%$, que se correlaciona con hallazgos previamente descritos de infección en sitio operatorio con rangos de $0.02 \%$ a $0.34 \% .^{(5,7,13-15)}$ Siendo la infección posquirúrgica importante en los pacientes sometidos a cirugía electiva, la infección severa que requiriere manejo hospitalario no se identificó en nuestra serie, lo que nos permite concluir que el riesgo general de infección tras una cirugía estética es bajo, al igual que la necesidad de servicios médicos que durante la pandemia son necesarios para atender a pacientes de COVID-19.

Según Keyes y col.(16) la mortalidad en cirugía ambulatoria es de 1 en 51.459 cirugías y Morello y col. ${ }^{(5)}$ reportan una mortalidad de 1 en 58.810 procedimientos $(0.0017 \%)$ y 2.02 fallecimientos por 100.000 procedimientos, la mayoría relacionados con embolismo pulmonar después de abdominoplastia. En nuestro estudio encontramos que en el periodo de 3 años se realizaron 1166 abdominoplastias y ningún paciente falleció por esta causa. La tasa de mortalidad en nuestro estudio fue de $1 / 13.411(0.007 \%)$ en una paciente que se sometió a cirugía combinada que incluyó liposucción, lipoinyección glútea, blefaroplastia y corrección de cicatriz. Durante estos 3 años se realizaron 4355 liposucciones. Luego del análisis de este caso en particular, no se identificó embolismo pulmonar, sobrecarga de líquidos o toxicidad por lidocaína que son condiciones implicadas en otros 
casos de fatalidad tras liposucción. ${ }^{(14)}$ Paredes Duque ${ }^{(17)}$ calculó que en Colombia, entre los años 2003 y 2005, se presentaron entre 180 y 200 fallecimientos asociados a liposucción para un estimado en el mismo periodo de 180.000 procedimientos. Según estas cifras se presentó 1 fallecimiento por cada 900 a 1.000 liposucciones, lo que contrasta con 1 fallecimiento por cada 47.417 procedimientos realizados en los Estados Unidos según reportes de la Sociedad Americana de Cirugía Plástica y Estética. En nuestro estudio se presentó 1 fallecimiento en 13.411 cirugías en un período de 3 años en el que se realizaron 4.355 liposucciones. En el análisis de este caso se identificaron la anemia aguda, shock hipovolémico y coagulopatía como factores asociados con el deceso.

La hemorragia es una de las causas más comunes de la inestabilidad hemodinámica en pacientes sometidas a liposucción. ${ }^{(14)}$ Del total de las complicaciones presentadas en nuestra serie en el periodo de 3 años 64 pacientes $(25.8 \%)$ presentaron anemia aguda, de los cuales $54(22.1 \%)$ requirieron transfusión por inestabilidad hemodinámica y 7 hospitalización para tratamiento y seguimiento. La mayoría de las hemotransfusiones fue instaurada de forma ambulatoria, sin embargo, algunos pacientes requirieron manejo hospitalario para el tratamiento de esta complicación, compitiendo por recursos hospitalarios reservados para los pacientes afectados por la COVID-19. De las 4.355 liposucciones realizadas en estos 3 años, 136 presentaron complicaciones para una tasa de $3.12 \%$ complicaciones asociadas a liposucción, de las que 58 fueron debidas a anemia aguda para una tasa de $1.33 \%$ en 3 años. Aun cuando el porcentaje de transfusiones es bajo son una causa frecuente de complicaciones, por lo cual es importante tener en cuenta medidas previamente definidas para minimizar la anemia posquirúrgica después de una liposucción, como son la realización de procedimientos únicos, corto tiempo quirúrgico, volúmenes bajos de lipoaspirado y adecuada infiltración con vasoconstrictores. De este modo consideramos que se podría reducir el número de pacientes con anemia, necesidad de transfusión, hospitalización y consumo de recursos médicos que pueden ser necesarios para la atención de pacientes de COVID-19.

De otro lado, en la práctica actual de la Cirugía Plástica Estética, es común la realización de varios procedimientos en un solo acto quirúrgico. Durante los 3 años de análisis encontramos que el $32.5 \%$ de las 13.411 cirugías realizadas en el periodo fueron combinadas y el $65 \%$ de las 244 cirugías que presentaron complicaciones fueron también combinadas. Dentro de los pacientes complicados con liposucción, el 89\% fueron cirugías combinadas. En general las cirugías combinadas se asocian a un mayor tiempo operatorio, sangrado y trauma quirúrgico, lo que explica el mayor número de complicaciones al asociar varios procedimientos en un solo acto quirúrgico. ${ }^{(18)}$ Harton y col. ${ }^{(4)}$ reportan una mortalidad asociada a liposucción como procedimiento único en el $0.0021 \%$ de los pacientes (1 por cada 47.415 cirugías), pero la mortalidad se incrementa significativamente cuando se tienen en cuenta cirugías múltiples. Según estos autores, al combinarla con procedimientos diferentes a abdominoplastia la mortalidad se incrementa al $0.0305 \%$ (1 por cada 7314 pacientes). Cuando la liposucción se acompaña de abdominoplastia u otros procedimientos la mortalidad se incrementa en 1 paciente por cada 3281 cirugías. Desde este punto de vista consideramos recomendable, con el fin de disminuir recursos hospitalarios posoperatorios, evitar la realización de procedimientos múltiples, pues es claro que al combinar cirugías en un solo acto anestésico se aumenta la morbi-mortalidad y la necesidad de recursos hospitalarios adicionales. En este mismo sentido sería recomendable realizar la liposucción como procedimiento único y no combinado, tal y como ya fue descrito. Actualmente y de acuerdo con varias recomendaciones, se ha asociado mayor tasa de complicaciones a los volúmenes de lipoaspirado alto, lo cual no fue analizado en nuestro estudio, sin embargo, en las publicaciones estudiadas, las liposucciones con aspirados inferiores al $1.500 \mathrm{ml}$ son consideradas procedimientos con menores tasas de complicaciones, mientras que lipoaspirados superiores a $3.000 \mathrm{ml}$ son considerados procedimientos quirúrgicos con mayor riesgo de complicaciones. Cuando las pérdidas sanguíneas exceden los $500 \mathrm{ml}$ o la duración de la cirugía es superior a 2 horas, la morbilidad y la mortalidad se incrementan significativamente. ${ }^{(18)}$ Según nuestros hallazgos, el promedio del tiempo quirúrgico de los pacientes que se complicaron fue de 200 minutos (percentil 25 de 113 minutos); es decir, las cirugías cortas, inferiores a 2 horas, presentaron pocas complicaciones y por lo tanto, mínimas posibilidades de requerir recursos hospitalarios. A pesar de que las liposucciones con estas características no están exentas de complicaciones, los datos recolectados soportan que de este modo sería posible disminuir significativamente el número de pacientes con complicaciones severas y la necesidad de recursos hospitalarios adicionales.

Entre las complicaciones anestésicas están descritas las relacionadas con el cuidado del monitoreo anestésico, de la anestesia general y las consideradas menores. Entre las más graves y con presentación más baja se encuentra el fallecimiento o daño cerebral del paciente, siendo éstas las que generan los mayores costos y repercusiones familiares y sociales. Las náuseas y el vómito son consideradas complicaciones asociadas a la anestesia y se presentaron con una frecuencia de entre el 10 al 80\%. 
Es un problema que se presenta cada día en los cuidados posperatorios, siendo bastante temidas por los pacientes e incrementando los costos del cuidado, retraso en el alta hospitalaria y en algunos casos el reingreso. La sobresedación o la falta de la misma, al igual que la inyección de anestésicos locales en lugares no indicados, se consideran como complicaciones asociadas a los anestésicos. ${ }^{(19)}$ En nuestro estudio identificamos 8 casos entre 248 complicaciones (3.2\%) de eventos adversos asociados a medicamentos, incluyendo las náuseas y el vómito. Sin embargo, consideramos que en este caso se presenta un subregistro de estos eventos porque en la mayoría de las ocasiones se consideran menores. Los casos registrados en nuestro estudio corresponden a pacientes que presentaron náusea y vómito persistente que obligaron a su permanencia por periodo prolongado en la sala de recuperación posquirúrgica o que les obligaron a reingresar; sin embargo, es relevante que este tipo de complicación no consumió recursos de unidades hospitalarias externas.

Otras complicaciones de la anestesia general incluyen el paro cardiaco, depresión respiratoria, broncoaspiración, despertares del paciente durante la anestesia, imposibilidad para retomar la consciencia, neuropatía periférica, reacciones alérgicas, fallo renal o hepático, mal funcionamiento de equipos y fallecimiento. La incidencia de broncoaspiración es de 1 en 3.216 actos anestésicos, siendo 4.3 veces más frecuente en cirugías urgentes. El $64 \%$ de los pacientes que broncoaspiran no desarrollan tos, sibilancias o disminución de la saturación de oxígeno en las siguientes 2 horas al evento, ni presentan secuelas respiratorias. La mitad de los pacientes restantes necesitan soporte respiratorio hasta 6 horas después del evento y $5 \%$ fallecieron por la insuficiencia respiratoria. En las 13.411 cirugías realizadas durante nuestro periodo de estudio se presentó 1 evento de broncoaspiración que requirió manejo intrahospitalario, y en total se presentaron 4 casos (1.6\%) con laringoespasmo y 10 casos (4\%) con edema agudo de pulmón y/o depresión respiratoria tras la anestesia. Además, 1 paciente con laceración faríngea y 1 paciente con isquemia cerebral transitoria. En general, todas estas complicaciones estuvieron asociadas a la anestesia general, por lo cual, en lo posible, el uso de anestesia regional o local podría disminuir en parte las complicaciones asociadas a la anestesia general en este tiempo de pandemia y disminuir la necesidad de recursos hospitalarios en el posoperatorio.

Las complicaciones asociadas a la mamoplastia de aumento son la cicatrización hipertrófica en el $6.3 \%$, hematoma en el $0.92 \%$ a $3 \%$ y seromas en la fase inmediata entre el $0 \%$ y el $1 \%$ y tardíos en el $1.68 \% .^{(20)}$ Los hematomas incrementan la tasa de infección y frecuentemente llevan a la contractura capsular. La mamoplas- tia de reducción con técnica de cicatriz vertical presenta una tasa de complicación global del 5.6\%. La complicación más frecuente es la dehiscencia superficial de herida quirúrgica, que ocurre en el $2.2 \%$ de los casos. La infección de la glándula mamaria ocurre en el $2.2 \%$ de los casos, hematomas en el $1.2 \%$, seromas en el $0.4 \%$ y necrosis grasa en el $0.8 \% .{ }^{(21)}$ En nuestro estudio, entre todos los eventos adversos presentados, el 31.9\% (79 de 248) correspondió a hematomas, de los cuales 44 se produjeron en mamoplastias de aumento con implantes. Es decir, la tasa total de hematomas entre todos los procedimientos realizados fue de 79 entre 13.411 pacientes operados en un periodo de 3 años, lo que corresponde a una tasa del $0.58 \%$. En general, las complicaciones asociadas a cirugía mamaria sin asociar otros procedimientos como liposucción presentan complicaciones que requieren ninguno o mínimos recursos hospitalarios tras la cirugía, sin impactar o competir con la población afectada por COVID-19 durante esta pandemia y que pudieran ser necesarios para salvar la vida del mayor número de afectados. La mayoría de estas complicaciones son resueltas en los centros de atención ambulatoria sin requerir hospitalización. Aunque el neumotórax intraoperatorio se ha descrito como una complicación de cirugía debido a procedimientos en torno a la pared torácica, como la obtención de injertos de costilla, cirugías de la pared torácica, colgajos musculares, o reconstrucción de la pared torácica, incluyendo la reconstrucción mamaria, Osborn y Stevenson ${ }^{(22)}$ reportaron 83 casos de neumotórax durante el aumento de senos y 19 casos fueron reportados por Keyes y col. ${ }^{(13)}$ En nuestra revisión encontramos 1 paciente que presentó neumotórax y requirió toracostomía y manejo hospitalario. Este tipo de complicación, aun cuando es inusual, en caso de presentarse durante la pandemia de la COVID-19 requerirá recursos hospitalarios que pueden ser necesarios para atender a esta población; sin embargo, la baja presentación de esta complicación no generará grandes alteraciones en caso de implementarse este tipo de cirugía. Por lo tanto, consideramos que la cirugía mamaria como procedimiento único sería susceptible de realizarse al reiniciar los servicios en tiempos de pandemia.

La lesión de estructuras intraabdominales y retroperitoneales se ha descrito como una complicación grave en los pacientes sometidos a liposucción, que se asemeja al trauma cortopunzante cuando la cánula penetra hasta estructuras profundas o en planos profundos. ${ }^{(14)}$ En nuestra serie identificamos 2 pacientes que requirieron laparotomía exploradora por este tipo de lesión. Aun cuando este tipo de reintervención es poco frecuente, en caso de presentarse requerirá manejo intrahospitalario, incluso de cuidado crítico. Por lo tanto, durante tiempos de pande- 
mia sería prudente la realización de la liposucción como procedimiento único, con volúmenes bajos de lipoaspirado (inferiores a 3 litros), con corto tiempo quirúrgico e incluso con anestesia local, como hemos mencionado previamente.

En general, identificamos en nuestro estudio que la mayoría de las complicaciones en Cirugía Estética son resueltas con manejo médico en el consultorio o de forma ambulatoria. El $82 \%$ de los eventos presentados fueron tratados de forma ambulatoria con seguimiento, recomendaciones, instrucciones y medicamentos, es decir, sin requerir recursos hospitalarios adicionales. Sin embargo, en el $18 \%$ de los casos complicados se requirió manejo hospitalario, siendo este el grupo de cirugías identificadas con el fin de reducir su práctica durante el período de reapertura pos-COVID-19. Adicionalmente, las principales comorbilidades que aumentan el riesgo de complicaciones posquirúrgicas y de pacientes infectados por COVID-19 son la malnutrición, obesidad, enfermedades del tejido conectivo, secuelas de radioterapia, trastornos vasculares, inmunodeficiencias, tabaquismo y enfermedades sistémicas mal controladas, como la diabetes mellitus y la hipertensión arterial. Se debería evitar operar a pacientes con este tipo de comorbilidades en este periodo de pandemia hasta que se cuente con una adecuada disponibilidad de recursos hospitalarios, debido a que este tipo de pacientes presentan mayor riesgo de sufrir complicaciones y competirían con los recursos disponibles para pacientes enfermos por COVID-19 o sería el grupo con presentación más grave de la enfermedad.

Finalmente, de los 44 pacientes que en nuestro estudio requirieron admisión hospitalaria (18\%) en el período de 3 años analizados, la gran mayoría fueron por cirugía combinada, liposucción y abdominoplastia. Por el contrario, las complicaciones después de cirugía mamaria y facial, incluyendo la ritidoplastia, permitieron el manejo del paciente en el centro de atención ambulatoria, especialmente para el drenaje de hematomas, requiriendo mínimos recursos hospitalarios adicionales, por lo que consideramos que la cirugía mamaria y facial podría realizarse durante la pandemia de la COVID-19.

La relevancia del presente análisis se sustenta en que las recomendaciones generadas no se basan en estudios poco comparables de diversas partes del mundo o en revisiones de la literatura médica concerniente a cada uno de los procedimientos descritos en Cirugía Plástica Estética, sino que surgen de una extensa base de datos local, de una sola institución, ambulatoria y no dependiente del sistema de salud, que incorpora todos las cirugías realizadas en un período definido pre-COVID, extrayendo datos aplicables a la realidad actual.

\section{Conclusiones}

Según el análisis realizado en nuestro estudio, teniendo en cuenta las cirugías que presentaron menor número de complicaciones en un centro ambulatorio en un período pre-COVID 19 y asumiendo el restablecimiento de los servicios de Cirugía Plástica en el contexto actual, las intervenciones de Cirugía Estética con menor posibilidad de consumir recursos hospitalarios externos y que no competirán con los recursos destinados a atender paciente afectados por COVID-19 incluyen procedimientos únicos y no combinados, cirugías con tiempos quirúrgicos cortos, idealmente inferiores a 2 horas, procedimientos bajo anestesia local y regional para disminuir las complicaciones asociadas a la anestesia general, cirugía mamaria y facial como procedimientos únicos.

\section{Dirección del autor}

Dr. Adolfo Alejandro López Rios

Calle 4 sur 43 a 195, Interior 121

Medellín, Colombia

Correo electrónico: aalejandro.lopez@udea.edu.co

\section{Bibliografía}

1. World Health Organization. Preguntas y respuestas sobre la enfermedad por coronavirus (COVID-19) [Internet]. 2020 [cited 2020 May 17]. p. 1. Available from: https://www.who.int/es/ emergencies/diseases/novel-coronavirus-2019/advice-for-public/q-a-coronaviruses

2. Rohrich RJ, White PF. Safety of outpatient surgery: Is mandatory accreditation of outpatient surgery centers enough? Vol. 107, Plast Rec Surg. 2001; 107(1): 189-192.

3. Keyes GR, Nahai F, Iverson RE, Singer R. Evidence-based medicine and data sharing in outpatient plastic surgery. Clin in Plast Surg 2013. 40(3):453-463.

4. Horton JB, Janis JE, Rohrich RJ. MOC-PSSM CME article: Patient safety in the office-based setting. Plast. Rec. Surg. 2008;122 (Suppl 3): 1-21.

5. Morelo DC, Colon GA, Fredricks S, Iverson RE, Singer R. Patient Safety in Accredited Office Surgical Facilities. Plast Rec Surg. 1997;99(6):1496-1500.

6. Warner MA, Shields SE, Chute CG. Major morbidity and mortality within 1 month of ambulatory surgery and anesthesia. JAMA J Am Med Assoc. 1993 22;2;70(12):1437- 1441.

7. Byrd HS, Barton FE, Orenstein HH, Rohrich RJ, Burns AJ, Hobar PC, et al. Safety and efficacy in an accredited outpatient plastic surgery facility: A review of 5316 consecutive cases. Plast Rec Surg, 2003; 112:6364-6371.

8. Teimourian B, Rogers 3rd WB. A National Survey of Complications Associated With Suction Lipectomy: A Comparative Study. Plast Rec Surg. 1989;84(4): 628-631.

9. Rubin JP, Matarasso A. Aesthetic surgery after massive weight loss. In: Saunders, Ed. Aesthetic surgery after massive weight loss. China: Elsevier; 2007. p. 196.

10. Roje Z, Roje Ž, Karanović N, Utrobičić I. Abdominoplasty complications: A comprehensive approach for the treatment of chronic seroma with pseudobursa. Aesth Plast Surg. 2006;30(5):611-615. 
11. Matarasso A. Awareness and avoidance of abdominoplasty complications. Aesth Surg J. 1997;17(4):256-261.

12. Chaouat M, Levan P, Lalanne B, Buisson T, Nicolau P, Mimoun M. Abdominal dermolipectomies: Early postoperative complications and long-term unfavorable results. Plast Rec Surg. 2000;106(7):1614-1618.

13. Keyes GR, Singer R, Iverson RE, McGuire M, Yates J, Gold A, et al. Analysis of outpatient surgery center safety using an internet-based quality improvement and peer review program. Plast Rec Surg 2004; 113:1760-1770.

14. Rao RB, Ely SF, Hoffman RS. Deaths related to liposuction. $N$ Engl J Med. 1999;340(19):1471-1475.

15. Natof HE. Complications Associated With Ambulatory Surgery. JAMA J Am Med Assoc. 1980;244(10):1116- 1118.

16. Keyes GR, Singer R, Iverson RE, McGuire M, Yates J, Gold A, et al. Mortality in outpatient surgery. Plast Rec Surg. 2008;122(1):245-250.
17. Paredes Duque JE. Factores de riesgo y complicaciones. In: Paredes Duque JE, Editor. Riesgos en cirugía plástica y estética. Cali, Colombia: Artes gráficas del Valle Ltda; 2005. Pp. 69-76.

18. Rohrich RJ, Mendez BM, Afrooz PN. An update on the safety and efficacy of outpatient plastic surgery: A review of 26,032 consecutive cases. Plast Rec Surg. 2018;141(4):902-908.

19. Goldberg M. Complications of Anesthesia for Ocular Surgery. Ophthalmol Clin North Am; 2006; 19: 293-307.

20. Collins JB, Verheyden $\mathbf{C N}$. Incidence of breast hematoma after placement of breast prostheses. Plast Rec Surg. 2012;129(3):413e-420e.

21. Lista F, Ahmad J. Vertical scar reduction mammaplasty: A 15year experience including a review of 250 consecutive cases. Vol. 117, Plast Rec Surg. 17(7): 2152-2165.

22. Osborn JM, Stevenson TR. Pneumothorax as a complication of breast augmentation. Vol. 116, Plast Rec Surg; 2005. $15 ; 116(4): 1122-1126$ 
